Du texte à la scène : langages du théâtre

\title{
Deux mises en scène de Richard II
}

\section{Valida Dragovitch}

\section{(2) OpenEdition \\ Journals}

Édition électronique

URL : http://journals.openedition.org/shakespeare/462

DOI : $10.4000 /$ shakespeare.462

ISSN : 2271-6424

Éditeur

Société Française Shakespeare

Édition imprimée

Date de publication : 1 novembre 1983

Pagination : 69-90

Référence électronique

Valida Dragovitch, "Deux mises en scène de Richard |/ 》, Actes des congrès de la Société française Shakespeare [En ligne], 4 | 1983, mis en ligne le 01 janvier 2007, consulté le 01 mai 2019. URL : http:// journals.openedition.org/shakespeare/462 ; DOI : 10.4000/shakespeare.462 
SOCIETE FRANÇAISE SHAKESPEARE

Actes du Congrès 1982

\section{DU TEXTE A LA SCENE : Langages du Théâtre}

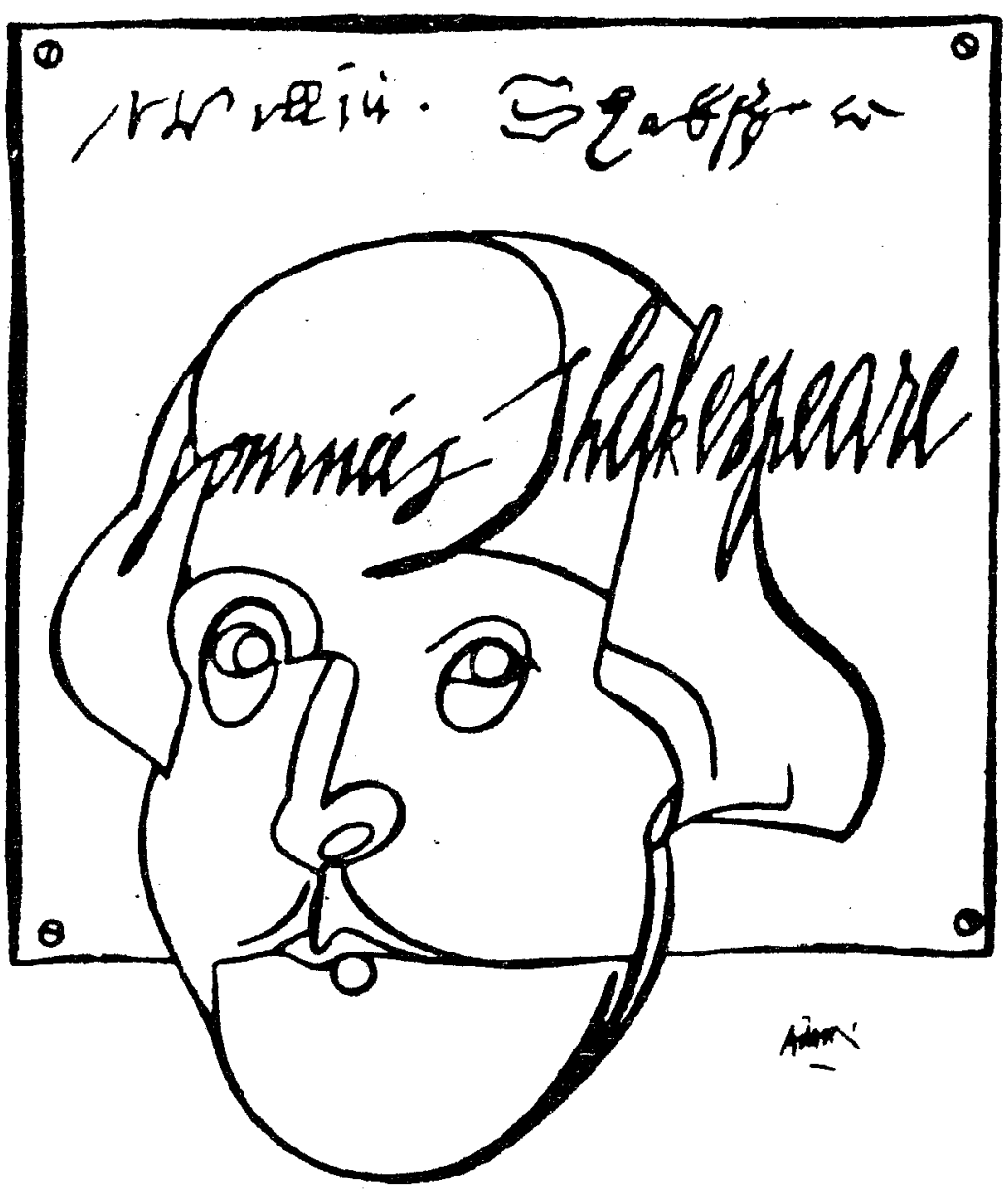

DiRecteur de la publication M.T. Jones - Davies

Publié avec le concours du Centre National de la Recherche Scientifique

JEAN TOUZOT Libraire - Editeur

38 , rue Saint-Sulpice 75278 PARIS CEDEX 061983 


\section{DEUX MISES EN SCENE DE RICHARD II}

Au début de cette année le public des deux grandes métropoles a eu la plaisir de voir la tragédie de Richard II de Shakespeare. La production de la Royal Shakespeare Company dirigée par Terry Hands avait débuté à Stratford en automne 1980 et le 13 mars 1982 donnait à Aldwich Theatre, à Londres, sa dernière représentation. Au même moment, à Paris, à la Cartoucherie de Vincennes, et sous la direction d'Ariane Mnouchkine, le Théâtre du Soleil présentait la même pièce depuis quatre mois.

J'ai toujours pensé que la représentation théâtrale d'une pièce est sa vraie naissance. C'est seulement à partir $\mathrm{du}$ moment où une pièce est jouée, où nous pouvons la voir et l'entendre, qu'elle existe réellement. Les créations de la Royal Shakespeare Company et du Théâtre du Soleil ont confirmé ma conviction. Leurs magnifiques mises en scène de Richard II sont les «mises-en vie» du texte de Shakespeare, aussi belles et aussi émouvantes que le texte.

J'ai été incitée à analyser parallèlement ces deux mises en scène par les très différentes méthodes que les deux compagnies théâtrales adoptent pour mettre en existence scénique l'oeuvre de Shakespeare. Mais, malgré les différences marquantes, ces deux mises en scène de Richard II sont l'une autant que l'autre fidèles à Shakespeare, dans la mesure où elles communiquent aux spectateurs le sens profond de la tragédie.

Il serait intéressant d'en faire une comparaison détaillée, mais elle exigerait plusieurs heures de développement. Je choisis de présenter ici séparément l'analyse de chacune de ces deux mises en scène, prenant la position d'un simple spectateur, d'un voyageur qui souhaite laisser un témoignage sur deux spectacles qui l'ont émerveillé.

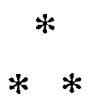




\section{RICHARD II DANS LA MISE EN SCENE DE TERRY HANDS}

La production de la Royal Shakespeare Company révèle une approche très respectueuse de Shakespeare, à la fois du texte et du sens de la pièce: Elle repose sur une structure équilibrée dans laquelle le jeu discipliné et traditionnel des acteurs et le décor, à la fois riche et sobre, se combinent harmonieusement. Si l'on considère aussi les costumes, l'éclairage et la musique composée pour le spectacle par Guy Woolfenden, on peut estimer que la représentation atteint presque la perfection.

Dans cette mise en scène tout le côté plastique et mobile de la scène met en évidence ce qui est contenu dans le texte. Le changement progressif des couleurs et des matières pour les costumes, les effets de lumière, le plateau de la scène lui-même qui, dans une grande partie de la pièce est incliné, ainsi que les objets utilisés, tout reflète à la fois la situation politique que Shakespeare présente dans Richard $I I$, et une atmosphère intérieure, spécifique pour chaque personnage, et particulièrement importante dans l'évolution de Richard II lui-même.

La scène, partagée en cinq allées, se prolonge dans le fond composé de cinq tableaux verticaux, qui représentent les personnages des nobles de chaque côté et celui du roi au milieu. Ces grands tableaux aux couleurs dorées font penser aux iconostases dans les églises orthodoxes. Ainsi, l'aspect même de la scène, au centre de laquelle se trouve le trône du roi, suggère l'existence d'un monde bien ordonné, organisé dans une hiérarchie typiquement élisabéthaine, soumis à la domination suprême de Dieu et ensuite à celle du roi. Les grands personnages peints évoquant les saints sur les icônes, créent en permanence une atmosphère religieuse, et transforment l'espace théâtral en un lieu sacré, habité par l'ordre et le rituel. En effet, le rituel est la ligne directrice de cette mise en scène. Tout se déroule avec une lenteur solennelle : chaque geste, posé et pesé, semble 
avoir été accompli ainsi depuis des siècles. L'équilibre de cette structure scénique repose sur une symétrie manifestée dans le décor autant que dans la disposition des acteurs. Cet équilibre est maintenu aussi par le côté très statique de la mise en scène, dans laquelle les mouvements sont réduits au maximum à l'exception de la scène avec la duchesse et le duc d'York au cinquième acte.

Cette structure ordonnée et équilibrée est très importante dans Richard II. C'est d'elle que Shakespeare part pour axer le développement tragique sur la destruction de cet ordre et de cet équilibre; dans Richard II, comme dans ses autres tragédies, c'est de cette destruction même qu'une nouvelle structure renaît. Pour rendre un tel déroulement évident, le metteur en scène donne une grande importance à la structure concrète et immédiatement perceptible du plateau. Celui-ci joue le rôle d'un support matériel qui aide à comprendre l'évolution intérieure de l'oeuvre dramatique.

Dès l'ouverture du premier acte on remarque un objet de grande importance symbolique - le cerf doré posé à côté du trône. Outre qu'il représente l'emblème royal du roi Richard II, le cerf évoque le thème de la chasse et place au centre du jeu l'opposition entre la cité et la forêt, entre la civilité et la sauvagerie. La loi protégeant le règne d'un roi reculera devant la force par laquelle l'au tre roi accèderá au trône. La présence du cerf doré sur la scène suggère ainsi la question fondamentale posée dans Richard II comme elle le sera dans Jules César : la déposition et le meurtre du roi - est-ce un sacrifice ou un sacrilège ? Terry Hands ne cherche pas à nous donner la réponse; son mérite est grand d'avoir suggéré la question.

Dans la troisième scène du premier acte, lorsque Richard interrompt le duel entre Bolingbroke et Mowbray, cette transgression de la part du roi est accompagnée par la rupture des cordes qui délimitaient l'espace du combat. Cette rupture traduit en image la cassure des liens qui définissent l'ordre de l'univers, l'ordre du pouvoir et l'ordre de la nature. En interrompant le combat, le roi 
ôte à Dieu le pouvoir suprême d'en décider l'issue. Ainsi, le roi s'oppose à Dieu. On le soupçonne d'être impliqué dans le meurtre d'un oncle et on le voit se moquer de la mort de l'autre, Jean de Gand. Les deux cousins, Bolingbroke et Richard, se haissent et la trahison prend la place de la loyauté. Le roi se comporte comme un tyran saisissant des biens qui ne lui appartiennent pas; ce même roi est déposé et finalement le sang du roi porte souillure à la propre terre du roi.

L'équilibre originel troublé, le renversement et le désordre qui s'installent dans le monde de Richard II sont reflétés dans le langage scénique par une grande inclinaison du plancher de la scène au troisième acte. Le sol stable se transforme en une pente sur laquelle marcher droit devient impossible et l'appréhension d'un glissement ou d'une chute s'éveille spontanément. C'est la scène du retour d'Irlande du roi Richard. Richard II interprété par Alan Howard, apparaît au sommet de la déclivité. Il est accompagné par l'évêque de Carlisle et le duc d'Aumerle. Faisant quelques pas en avant, il s'agenouille pour toucher de la main la terre qu'il est en train de saluer. Le costume qu'Alan Howard porte à ce moment-là fait partie de ce langage plastique qui transmet une image du texte à la scène.

Ce costume, composé d'une tunique et d'un pantalon, est en tissu mat, de couleur jaune pâle. Les manches ainsi que la cape se terminent par des pointes découpées dans le même tissu, pendant jusqu'au sol. Lorsque Alan Howard apparaît au sommet de la scène oblique, son costume fait penser au soleil, mais à un soleil terne, quittant le zénith, tourné vers l'ouest. Ses rayons n'émanent pas de lui, élouissants, mais tombent, obscurcis par la mélancolie du crépuscule.

$\mathrm{Au}$ fur et à mesure que les événements se poursuivent, le roi Richard émerge identique à Phaéton auquel il se compare :

Down, down I come, like glist'ring Phaeton, Wanting the manage of unruly jades. 
(III, iii, 178-179)

Le costume que je viens de décrire accentue l'importance de ces lignes derrière lesquelles se cache le malheureux fils d'Hélios, précipité dans sa perte, non par la faute des chevaux déchaînés, mais par son inexpérience dans la conduite du char céleste. Ne pouvant pas contrôler le galop enflammé avec la force de son père, Phaéton provoque lui-même sa chute. Cette interprétation figurant explicitement dans les Métamorphoses d'Ovide, suggérée dans la mise en scène de Terry Hands par le vêtement du roi, attire l'attention du public sur le caractère déviant du règne de Richard II. Comme Phaéton, il sera précipité des hauteurs à cause de sa propre incompétence à conduire le char royal.

Un autre costume porté par Alan Howard est presque aussi significatif. Lorsque, dans la première scène du quatrième acte, il prononce ces mots:

O that were a mockery king of snow, Standing before the sun of Bolingbroke, To melt myself away in water drops!

(IV.i. 260-262)

son costume est tout blanc.

Le lien entre les principaux costumes du roi est remarquable. Celui que le roi porte au premier acte est en tissu doré et suggère la splendeur du soleil de midi. Le costume suivant évoque l'image d'un soleil moins éblouissant, dont la brillance est affaiblie lors de son glissement vers 1'horizon: Richard est d'abord un soleil qui par sa hauteur domine le monde, par sa lumière l'éclaire et par sa chaleur lui inspire la vie; ensuite il se transforme en une figure de neige. Il se sent lui-même proche de cette matière blanche et froide qui évoque l'hiver, le silence, la mort. Le roi s'est transformé en son contraire - il ne tient plus le sceptre dans sa main, ne porte plus la couronne, ne monte plus sur le trône - de même que la Pythie ne serait pas la Pythie aux pouvoirs prophétiques sans le trépied et sans les branches de laurier, 
démuni des insignes royaux le roi n'est plus roi.

La bouleversante scène de la déposition, ainsi que la scène du miroir, apportent aux vers de Shakespeare une nouvelle dimension. Avant de tendre la couronne à Bolingbroke, le roi la pose sur sa tête et l'enlève aussitôt. Ce geste bref précède l'offre séduisante, «Here, cousin, seize the crown». Mais la couronne n'est pas tout de suite abandonnée à la main qui l'a saisie. Pendant quelques instants les mains des deux cousins restent figées sur la couronne placée entre eux. Dans une tension croissante on se demande si c'est la couronne qui maintient debout les deux hommes; si la couronne a tendance à s'élever et à les élever avec elle, ou si, au contraire, elle les écrase tous les deux, chargeant leurs mains et opprimant leurs têtes d'un poids insoutenable.

Here, cousin,

On this side my hand, and on that side thine.

Now is this golden crown like a deep well

That owes two buckets, filling one another,

The emptier ever dancing in the air,

The other down, unseen, and full of water.

That bucket down and full of tears am I,

Drinking my griefs, whilst you mount up on

high. (IV.i. 182-189)

Deux mouvements opposés sont suggérés par ces vers : l'élévation et l'engloutissement. Shakespeare fait voir à Richard la couronne comme un puits. De même qu'un puits, la couronne permet ces deux mouvements contraires. Comme l'eau d'un puits elle élève et nourrit. Mais elle peut aussi devenir un abîme, un gouffre dévorant dont on ne sort plus. La même couronne qui fera la déchéance de Richard, fera l'élévation de Bolingbroke.

La mise en scène de Terry Hands met en relief cette double fonction de la couronne, et suivant la métaphore de Shakespeare, fait qu'elle paraît au public à la fois nourricière et meurtrière.

$\mathrm{Si}$ Shakespeare fait suivre la scène de la déposition par la scène du miroir, c'est probablement pour nous 
rappeler que le gain ainsi que la perte du pouvoir ne sont que des états passagers, incertains, illusoires.

Le nouveau roi est installé sur le trône. Le roi déchu est assis sur la marche menant au trône qui, il y a à peine un instant, était le sien. Le miroir qu'il réclame lui est apporté, non par un serviteur comme l'indique le texte, mais par le duc d'York lui-même. Alan Howard prend le miroir ovale dans sa main. Il lit dans le reflet du roi déchu le reflet des choses qui, elles aussi, ne sont que des apparences. Immobile, assis en bas du trône, Alan Howard fixe le miroir. Il ne détruit pas la surface trompeuse en la jetant par terre, il la brise avec son poing. La glace brillante, qui semblait s'être imprégnée du visage, s'envole sur la scène en mille éclats. Le cadre vide dans la main, son regard plonge à travers ce cercle comme dans les profondeurs d'un puits, comme dans le néant d'une couronne perdue. Alan Howard apporte une intensité particulière à cette scène émouvante. Il nous fait suivre le regard du roi qui tombe dans le vide. Il nous fait sentir son propre anéantissement : «Ay, no; no, ay; for I must nothing be». A travers le miroir brisé il nous conduit jusqu'au néant dont il nous parle plus tard :

But whate'er I be,

Nor I, nor any man that but man is,

With nothing shall be pleas'd till he be eas'd

With being nothing.

$$
\text { (V.v. 38-41) }
$$

Son regard, pendant un long moment plongé dans le cadre vide, nous force à le suivre dans sa contemplation de la non-existence, dans sa rencontre avec la mort. Mais on est brusquement ramené à la réalité par la voix de Bolingbroke : «The shadow of your sorrow hath destroy'd/ The shadow of your face» (IV. i. 292-293). «L'ombre du visage» incite le roi à développer une nouvelle métaphore. En reprenant les paroles de Bolingbroke il est debout, il porte à son visage un tissu bleu-ciel.

The shadow of my sorrow ? ha, let's see 'Tis very true, my grief lies all within, 
And these external manners of lament

Are merely shadows to the unseen grief

That swells with silence in the tortur'd soul.

There lies the substance.

(IV.i. 294-299)

Ayant pressé le tissu sur son front, Howard le tient devant lui, l'observant attentivement : son visage n'y a pas laissé d'ombre. D'un geste de colère et d'amertume il le jette par terre. Richard n'est pas le Christ. Sa souffrance ne fait pas de lui une victime innocente. Ainsi, il faut continuer à voir Richard II comme Phaéton qui, ayant commis une erreur, ne put pas être sauvé par le Soleil lui-même.

Le texte imprégné des métaphores par lesquelles Shakespeare dépeint dans Richard II les différents stades de la transformation d'un monarque en un «non monarque» est transmis sur la scène par nombre de costumes différents.

La scène et les éclairages suivent une progression semblable à celle des costumes. Le sacré, la cérémonie, l'ordre et la puissance, sont rendus au premier acte par une scène lumineuse, des matières riches, des couleurs dorées, une parfaite symétrie de mouvements dessinés sur un fond à caractère religieux. La scène est assombrie par des tissus de velours et des couleurs d'automne lorsque Bolingbroke accède au trône. Il interrompt la lignée des rois angevins, sa révolte provoque un renversement des valeurs - ainsi le clair devient l'obscur, l'or est remplacé par le velours, et la scène, précédemment continue, est partagée en deux. Derrière un rideau entrouvert, Bolingbroke, assis à son bureau, semblable aux marchands comptant leur fortune, considère les affaires de l'Etat. Les nuances dans les couleurs et dans les éclairages utilisés vont de pair avec la politique de Bolingbroke qui n'est ni nette, ni éclatante, mais nuancée et ambiguë.

Après la scène de la séparation du roi déposé et de sa reine, intervient l'événement qui, dans la mise en scène de Terry Hands, est exploité conformément au texte, d'une manière comique. C'est la dispute du duc et de la duchesse d'York au sujet de leur fils Aumerle. Leur 
querelle, leur course affolée jusqu'au palais royal, leur plaidoirie devant le roi, provoquent le rire du public et font oublier l'angoisse qui provient du danger de mort qui menace Aumerle.

Ce danger dont on a pu rire, car il fut écarté, est suivi par un danger plus sombre et plus menaçant lorsque Exton prononce sa détermination de prouver son amitié au nouveau roi en le libérant de celui dont il occupe le trône.

La scène de la prison est l'une des plus impressionnantes. Le plancher est encore une fois incliné, mais cette fois-ci un plafond suspendu au-dessus de la scène est incliné lui aussi, de façon à créer une perspective profonde, portant le regard très loin et donnant l'illusion d'un horizon rapproché - comme si le ciel était descendu et pesait lourdement sur la terre, comme si la terre s'était élevée, guerrière, pour offenser le ciel. De cette ouverture étroite, au fond et au sommet de la scène, arrive le roi déposé. Il traîne derrière lui une chaîne longue et lourde. Il descend vers l'avant de la scène. Là, d'un geste violent, il lance la chaîne autour de lui, formant ainsi un cercle, dans lequel il entre, au milieu duquel il s'assied.

Cette scène évoque l'idée que Richard construit lui-même sa prison, provoque luị-même son isolement. Huit hommes arrivent pour le tuer. Sa vie se termine dans une lutte acharnée où personne ne vient le secourir. Il est abandonné à lui-même et meurt comme un cerf déchiqueté par les chiens.

La mise en scène de Terry Hands présente la fin de la tragédie de Richard II en rappelant certains thèmes évoqués au début. La chasse et la sauvagerie s'accomplissent dans l'assassinat de Richard. Mais l'ordre et la cérémonie sont rétablis. Pendant que le roi Henry IV annonce sa décision d'aller en Terre Sainte pour purger sa souillure, le chant du kyrie accompagne le départ solennel des courtisans emportant le corps du roi assassiné. L'équilibre, qui au début de la pièce est bouleversé par l'interruption des rites de chevalerie, est retrouvé dans les rites funéraires, 
dans le deuil ordonné pour pleurer la mort du roi Richard II.

\author{
* \\ * *

\section{RICHARD II dans la mise en scène d'Ariane Mnouchkine}

A part un praticable posé au centre et un panneau au fond, la vaste scène de la Cartoucherie de Vincennes n'est parée d'aucun décor. A droite de la scène un grand nombre d'instruments de musique attendent la personne qui viendra les animer. Jean-Jacques Lemêtre arrive presque inaperçu et le murmure dans la salle meurt avec les premiers coups de baguette qui font résonner la timbale à un rythme qui s'accélère. Dans ce mouvement rapide le roi Richard et sa cour arrivent sur la scène. Leur venue semble portée par le vent. Ils tournent autour de la scène comme dans une envolée, et s'arrêtent au moment où Richard, d'un saut qui ne laisse paraitre aucun effort, monte sur le praticable. C'est le trône sur lequel le roi reste debout. C'est le piédestal d'où le roi salue ses sujets. Il tient en position verticale son bâton royal, qu'il montre à tous, tournant lentement et fixant du regard chacun de ceux qui se trouvent face à lui. A ce moment même, Georges Bigot change les spectateurs en sujets du roi Richard II, qu'il vient de devenir. Son geste et son regard agissent sur le public comme un sort qui, au cours du spectacle, ne cesse de se renforcer.

De son trône, de son piédestal, le roi questionne le duc de Lancastre et les deux combattants sur les causes de leur conflit. Sa voix sonore et impérieuse retentit dans l'espace, pareille au torrent descendant de la montagne. Son regard a la force de l'éclair. Nous assistons à plus qu'une cérémonie. Nous sommes imperceptiblement portés en un temps presque mythique, où le souverain fait un avec le dieu. C'est lui qui exécute sa volonté auprès 
des mortels, c'est lui qui incarne sa puissance dans le monde terrestre.

$\mathrm{Au}$ coeur du roulement de la percussion, Bolingbroke et Mowbray s'accusent réciproquement, jetant à terre leurs gages et jurant leur loyauté et leur honneur au roi et à Dieu. Mais au moment où les rites de chevalerie devraient se dérouler, où le courage devrait présider le combat décisif, les adversaires terrifiants arrivent euxmêmes terrifiés et tremblants. Ce tournant inattendu est d'autant plus surprenant qu'avec le roi arrivent sur la scène les bouffons, criant, sautant, grimaçant; et la cour, que l'on voyait imposante et majestueuse, prend le visage d'un cirque. Au moment où nous sommes sur le point de croire à la tragédie, celle-ci se transforme en bouffonnerie et le rituel se transforme en sa propre caricature.

Pourtant, dans cette scène turbulente et en apparence comique, se trouve le noyau de la tragédie de Richard II. La cérémonie s'est transformée en bouffonnerie, les ardents chèvaliers en lâches. C'est le renversement qui appréhende un autre renversement. Avec le rire et l'angoisse nous pressentons qu'une autre métamorphose va se produire celle du roi lui-même. L'humeur changeante de la fortune, la hauteur inaccessible et périlleuse d'un monarque sont inscrites dans cette scène. Les contrastes manifestés entre le rituel et le burlesque, entre le sacré et le profane, entre l'obligation de vaillance et la réalité de la peur, incitent à penser au caractère dualiste de la fonction royale dont Richard parle lui-même à son retour d'Irlande, lorsqu'une totale désillusion l'envahit :

... Que personne ne parle de réconfort, Parlons de tombes, de larves et d'épitaphes, Que la poussière soit notre papier, avec la pluie des yeux

Ecrivons la douleur sur le sein de la terre, Choisissons des notaires et parlons testaments. Et pourtant non. Car que pouvons-nous léguer, Si ce n'est à la terre léguer nos corps déchus? Nos domaines, nos vies, tout est à Bolingbroke 
Et rien que nous puissions dire nôtre hormis

Et cette petite croûte de terre stérile

la mort, Dont comme avec de la pâte on couvrira nos os.

$\mathrm{Au}$ nom du ciel, asseyons-nous à terre

Et disons les tristes histoires de la mort des Rois:

Comment les uns ont été déposés, d'autres tués

D'autres hantés par les spectres de ceux qu'ils

à la guerre, avaient déposés,

D'autres empoisonnés par leurs femmes, d'autres

Tous assassinés. Car sous la couronne égorgés en dormant,

Qui enserre les tempes d'un Roi mortel

La mort tient sa cour. La bouffonne se tient là, Moquant la royauté, ricanant de sa pompe.

Elle lui octroie un petit théâtre

Pour jouer au monarque, être craint et tuer

Elle l'imbibe de vaine suffisance, d'un regard.

Comme si cette chair qui remparde notre âme Etait un imprenable acier : puis s'étant bien

Elle veut en finir, et avec une petite épingle amusée

Elle perce le rempart et adieu Roi.

Couvrez-vous. Ne raillez pas d'un hommage

Ce qui n'est que chair et os, solennel

Jetez au loin le respect, la tradition, l'étiquette, l'allégeance et le cérémonial,

Car vous m'avez confondu depuis toujours.

Je vis de pain comme vous, je ressens le besoin, J'éprouve la douleur, et j'ai besoin d'amis.

Asservi de la sorte, comment pouvez-vous dire que je suis Roi ? ${ }^{1}$

La nature double de l'existence d'un roi est le thème qui, dans la mise en scène d'Ariane Mnouchkine, guide le développement tragique de la pièce. Georges Bigot, incar- 
nant Richard II, nous fait sentir profondément la coexistence paradoxale du roi et de l'homme dans un seul corps.

Lorsqu'il lance son bâton royal pour interrompre le duel, Georges Bigot imprègne ce geste d'une intensité inouie qui exprime la contradiction fondamentale de son état. Cet acte nous fait comprendre que sa fonction politique est dérangée par le facteur humain qui le pousse à annuler lui-même ce qu'il est en train de signifier. En arrêtant le duel, Richard récuse la volonté de Dieu comme unique volonté supérieure à la sienne; c'est par sa propre volonté qu'il est roi. En s'opposant à Dieu il cherche à s'attribuer une puissance absolue; en réalité, il détruit par ce geste sa puissance de roi, puisqu'il la soumet à sa volonté d'homme.

La même dualité est visible dans le comportement de Richard envers son oncle. En tant que roi, Richard se permet de parler orgueilleusement et vertement au duc de Gand mourant. Mais, brusquement, en s'asseyant à côté de son oncle et en posant sa tête sur les genoux du vieil homme, il manifeste une tendresse d'enfant, une fragilité d'homme seul et sans soutien.

Pendant la pièce, Georges Bigot nous rappelle constamment cette dualité conflictuelle, qui pourtant ne demeure pas statique. Grâce à cet acteur, dont la force et la'sensibilité sont aussi grandes que le talent, nous pouvons suivre l'évolution de Richard II, au cours de laquelle la destruction du roi en Richard fait que l'homme en Richard peut grandir. Dans cette mise en scène, Richard II n'est pas bloqué dans son esprit enfantin et capricieux. Alors qu'Alan Howard mène l'évolution de Richard jusqu'à la désillusion totale, mais par la suite la fige dans une amertume qui ne lui donne du monde qu'une vision cynique, dépourvue d'amour et de compréhension, Georges Bigot sort Richard de son monde d'enfance, et son cynisme se transforme en une profonde souffrance d'homme adulte. D'un désastre politique, d'une chute, d'une mort, Georges Bigot fait émerger Richard comme un être plus beau, plus grand et plus fort, capable de s'élever 
vers les plus hauts sommets de l'existence humaine. Georges Bigot ne projette pas à travers Richard II l'image de Phaéton; il nous suggère plutôt celle du Phénix.

Le Richard II d'Ariane Mnouchkine est joué sur un rythme très vif, avec une énergie immense, libérée par les acteurs dans leur abandon total au mouvement que leur impose le texte de Shakespeare. Ce texte, ils le comprennent, ils le vivent et nous le font vivre.

Cette mise en scène de Richard II emploie tous les moyens d'expression : la musique, basée sur les rythmes des pays asiatiques, mais aussi africains; les gestes, stylisés comme dans une danse rituelle; les lumières, des plus éclatantes jusqu'aux spots n'éclairant que les visages des acteurs entourés de nuit; les couleurs, reflétant symboliquement $\mathrm{du}$ fond de la scène chaque nouvelle situation dramatique.

C'est en éveillant toute la sensibilité des spectateurs et en exigeant d'eux une réceptivité totale et active qu'Ariane Mnouchkine a traduit et a mis en scène Richard II de Shakespeare. Elle prouve que le langage du théâtre est illimité et que c'est en puisant dans sa richesse et dans sa variété, que l'on peut rendre vivant un texte écrit.

Les éléments du théâtre japonais figurant dans cette mise en scène sont nombreux. Nous remarquons les gestes qui rappellent les gestes typiques de l'étonnement, de la contestation et de la peur dans le kabuki. Les serviteurs habillés en noir et voilés font penser aux kuromaku - les manipulateurs de poupées dans le bunraku. L'articulation, ainsi que le timbre de voix que l'on entend en particulier chez les ducs d'York et de Lancastre évoquent le jôrurt, le récitatif accompagné de musique dans le bunraku ou dans le kabuki. Les masques portés par certains acteurs peuvent être comparés aux masques utilisés dans le nô, de même que la scène burlesque des jardiniers pourrait correspondre à la pièce comique en un acte, kyôgen, figurant dans le théâtre nô et censée égayer son atmosphère profonde et mystérieuse. Lorsque l'on observe les 
gestes rituels et cérémonieux du roi Richard II, on peut même faire un rapprochement avec le bugaku, la forme la plus ancienne du thêatre japonais, dérivée des cérémonies rituelles du shintô.

Ces correspondances japonaises prêtent le flanc à diverses critiques, mais elles sont justifiables, si on compare l'époque de Richard II en Angleterre avec la fin de l'époque Héian au Japon, la guerre des Roses avec les conflits acharnés des clans Genji et Heiké, et surtout si on remarque le parallèle entre le théâtre élisabéthain et le $k a b u k i$, dont se rapproche le plus cette spectaculaire et grandiose mise en scène de Richard II. La création du Théâtre du Soleil pourrait sans doute trouver un grand appui dans l'étude du Professeur Leonard Pronko, Kabuki and the Elizabethan Theatre ${ }^{2}$, ainsi que dans le remarquable ouvrage du Professeur Minoru Fujita, Pageantry and Spectacle in Shakespeare ${ }^{3}$ dans lequel il confirme la correspondance entre le théâtre élisabéthain et le kabuki:

The study of Shakespeare's original stagecraft in the light of contemporary royal and civic spectacles is especially profitable in that the latter is as rich in colour and motion, dance and music, and elements pleasing to the eye, as Kabuki is. Like Kabuki, pageant stages were remarkable for their tableau effects, stylization, ceremony, symbolism, and formalism, or, in a word, «spectacle».(...) Certainly the comparison and contrast of the Elizabethan drama with Kabuki is an interesting and attractive subject. Between the two theatre traditions there are certain parallels in historical background and certain striking similarities in technique. Professor Pronko suggests the merit of the study of Kabuki in reconstructing a more authentic Elizabethan performance. It is true that Kabuki still carries on a living theatrical tradition, which arose from the historical conditions somewhat similar to those of Tudor 
England, and which exhibited astonishing similarities to much of what seems to have taken place on the Elizabethan stage three or four hundred years ago.

Mais le plus important, c'est de souligner que le théâtre japonais n'a servi au Théâtre du Soleil que d'inspiration. Il la développe d'une manière très libre et très créative, ouvrant des horizons nouveaux au théâtre contemporain français. Le Théâtre du Soleil accède à une parfaite authenticité et originalité dans le jeu des acteurs, ainsi que dans les costumes, la musique et le décor.

Ce décor, que j'ai mentionné plus haut, est une peinture sur tissu, qui au cours de la représentation change dix fois. Le spectacle s'ouvre sur un fond rouge et doré. Bolingbroke et Mowbray s'affrontent devant un panneau tout rouge, tandis que la mort du Duc de Gand semble descendre sur la scène en or et en blanc. Le retour de Bolingbroke en Angleterre est signalé par un soleil levant, émergeant derrière une ligne d'horizon inclinée et situé sur le côté droit du panneau. Le panneau suivant, qui accompagne le retour du roi Richard, est, lui, parfaitement symétrique. Ainsi le soleil de Richard est le soleil couchant, situé à gauche, là où on représente l'ouest. A la fin de la deuxième scène du troisième acte, le roi, dans le désespoir et l'amertume déchire son ciel de nuit pour laisser entrer sur la scène le soleil d'une brillance éblouissante qui éclairera le grand jour de Bolingbroke.

A son retour en Angleterre, Bolingbroke est accueilli par Northumberland. Les deux nobles, interprétés brillamment par Cyrille Bosc et Maurice Durozier, arrivent sur la scène à cheval. Il s'agit là d'une illusion extrêmement convaincante, produite par la démarche des acteurs, dont les gestes évoquent à la fois des cavaliers et leurs chevaux. Cette sorte de chorégraphie est particulièrement impressionnante lorsque d'autres nobles se joignent à eux. Ces cavaliers téméraires font émerger devant nos yeux toute une armée galopant le long des «chemins rudes et inégaux» des «hautes collines» du Gloucestershire. La force grandit 
aux côtés de Bolingbroke. Mais, la crainte qu'inspirent ces guerriers se voit ajouter une touche d'humour - JeanPierre Marry, dans le rôle de Henry Percy, est déjà dans la peau de Hotspur, et avec une imagination effervescente nous le fait remarquer.

Quant au roi revenant d'Irlande, il ne blesse pas le sein de sa terre par une cavalerie rebelle.

Terre chérie, de cette main je te salue,

Toi qu'ont blessée les sabots des chevaux

rebelles.

Comme une mère après un long départ retrouvant son enfant

Mélange tendrement les larmes et les sourires, Ainsi moi, pleurant en souriant, je te salue

ma terre

Et de mes mains royales je te rends grâce.

(III. ii. 5-10)

Georges Bigot parle à la terre en la caressant. Elle est cette mère mythique qui s'ouvre pour laisser revenir à elle son fils, pour laisser retourner en elle son roi. Le lien profond par lequel Shakespeare unit la terre et le roi est accompli sur la scène par un acte d'amour. C'est l'acte dans lequel la scène austère devient la Terre, la femelle primordiale, Gaïa , qui donnera naissance aux Titans.

Cette terre aura du sentiment et avant que son

roi légitime

Ne chancelle sous les perfides armes de la rébellion,

Il s'avèrera que ces cailloux sont ses sol dats en armes.

(III.ii. 23-25)

confirme Richard. Georges Bigot nous en persuade. Il nous emmène au temps d'Uranus et nous fait témoins de son union avec la Terre, ainsi que de son horrible destruction. Le roi Richard est détrôné par l'homme de son propre sang qui n'est que son sujet.

Ayant abandonné sa couronne et son sceptre, le roi enlève une par une les pièces de son somptueux vêtement blanc. Lorsque ses habits tombent par terre, nous croyons 
voir réellement ce roi de neige auquel il se compare, fondre sous le soleil de Bolingbroke. Georges Bigot est seul à ce moment-là. Il se tourne vers le public qui doit alors devenir son dernier refuge et son unique complice. Et nous le devenons. Lorsqu'il parle, agenouillé sur le bord de la scène, entouré des soieries blanches qui revêtaient son corps royal, entouré d'éclats éparpillés de sa splendeur brisée dans le reflet d'un miroir, regardant à travers un voile de larmes chacun de nous, quelque chose nous lie à ce roi tragique que soudain nous croyons connaître et comprendre. Jouée devant un fond pourpre, cette scène nous laisse un goût âpre, avec l'image de la neige fondant dans un bain de sang. La scène de la déposition ne nous épargne aucune douleur. Nous souffrons avec le roi, physiquement ainsi que moralement, et dans les termes d'Aristote, cette scène représente une vraie catharsis.

A la fin du quatrième acte, la tragédie de Richard II atteint son sommet. Mais Ariane Mnouchkine et ses acteurs poursuivent la tragédie de Shakespeare avec une intensité dramatique inchangée.

Dépouillé de son sceptre royal et de sa couronne, Richard doit se séparer aussi de sa reine. La rupture forcée des liens entre le roi et sa terre est juxtaposée à la séparation du roi et de sa femme, Isabelle, à laquelle Shakespeare accorde intentionnellement l'âge adulte.

D'un fond sombre que définit le visage funeste de la lune, le roi déchu s'avance enchaîné. Loin derrière lui, six hommes en noir tiennent les chaînes qui l'oppriment. La fureur et la douleur qu'il éprouve lui donnent une force immense et lorsqu'il tire violemment ses chaînes, il fait tomber à genoux ses sinistres gardiens. Richard se transforme en ce lion blessé, en ce roi des bêtes sauvages qu'évoque Isabelle. Mais en même temps, avec une esthétique rare, et une sensualité raffinée, Georges Bigot et Odile Cointepas nous font découvrir un Richard profondément humain, doué d'amour et aimé. Partagés entre la violence et la tendresse, entre la force et la faiblesse, entre l'amour et l'amertume, les deux acteurs 
nous communiquent le déchirement affectif et moral du couple royal.

La deuxième scène du cinquième acte, la dispute entre le duc et la duchesse d'York, qui suit la scène des adieux, est, dans la mise en scène d'Ariane Mnouchkine complètement omise. Ayant placé l'accent comique au milieu de la pièce, présentant la scène des jardiniers sous un aspect burlesque - la scène qui, à Aldwich Theatre, était jouée sur un ton sérieux - Ariane Mnouchkine n'exploite aucune des possibilités d'humour qu'offre la querelle familiale au cinquième acte. Au contraire, elle exclut totalement et cette scène et la duchesse d'York, et abrège la scène suivante d'une manière radicale ce qui, malheureusement, provoque quelques confusions au sujet du duc d'Aumerle, qui, agenouillé devant le roi Henry IV, lui adresse les paroles que normalement devrait prononcer sa mère. Mais ces quelques défauts mis à part, ce raccourcissement permet à l'impression créée par la scène des adieux d'être toujours vivante lorsque la grille de la prison de Richard apparaît devant nos yeux.

Derrière cette grille, celui qui était roi tourne son regard une fois de plus vers l'auditoire muet. Sa voix ne résonne plus comme le tonnerre, mais arrive vers nous comme une brume. Avec sa voix, les barreaux de sa cage semblent se dissoudre et la prison de Richard, devenue immense et oppressante, nous envahit et nous aspire. Richard la peuple de ses pensées. Mais ne sommes-nous pas les pensées mêmes de Richard, produits par son imagination pour vivre avec lui sa chute, sa torture, sa mort?

Plusieurs fois, Georges Bigot s'élève à des hauteurs inaccessibles avec le roi Richard. Plusieurs fois, il tombe et se relève à nouveau, jusqu'au moment où il est précipité «comme l'éclatant Phaéton» perdant son chariot de feu, son titre, sa vie. Mais Georges Bigot fait du Richard déchu un perdant dont la chute se transforme en un nouvel envol. Ce dernier envol est sans pareil. C'est dans sa cage étroite, presque nu, démuni de ses pouvoirs terrestres et de ses possessions, que Richard atteint sa plus 
grande hauteur et sa plus grande force.

A travers la grille de sa cage Richard est poignardé à mort. Mais, avant de mourir, il se défend avec l'acharnement du lion piégé. Deux de ses bourreaux restent accrochés à la cage, mourant en même temps que leur victime.

Dans la scène de la prison, Georges Bigot fait disparaître toutes les barrières. Nous rendant témoins de la mort du héros tragique, il nous lie directement à Shakespeare.

La scène finale est imprégnée d'une solitude infinie dans laquelle les deux rois arrivent enfin à se rencontrer. Sur un fond noir et doré se dessine le trône royal sur lequel Henry IV berce dans ses bras le corps de Richard assassiné. Brusquement, nous découvrons dans cette image la Pietà Rondanini, nous rappelant l'avertissement prophétique de Michel-Ange : «On n'imagine pas combien de sang cela coûte».

L'instant suivant Henry IV laisse tomber par terre le corps de Richard et s'allonge sur son trône, sur le praticable qui devient à ce moment-là un lit de mort. Les deux rois sont superposés : l'un n'est plus qu'un corps mort, l'autre est encore capable de porter la couronne.

Ariane Mnouchkine finit la tragédie de Richard II sur une ouverture au thème célèbre d'Henry $I V:$ «Uneasy lies the head that wears the crown». 


\section{NOTES}

1. Traduction Ariane Mnouchkine, Solin, Paris, 1982, III.i.pp. $79-80$.

2. Leonard C. Pronko «Kabuki and the Elizabethan Theatre», Total Theatre, ed. E.T.Kirby, New York, 1969.

3. Minoru Fujita, Pageantry and Spectacle in Shakespeare, Sambi Printing Company, Tokyo 1982, p. iv, v. 